\title{
Discovery of Associated Absorption Lines in an X-Ray Warm Absorber:[ITAL]Hubble Space Telescope[/ITAL] Observations of PG 1114+445
}

\section{Citation}

Mathur, Smita, Belinda Wilkes, and Martin Elvis. 1998. "Discovery of Associated Absorption Lines in an X-Ray Warm Absorber:[ITAL]Hubble Space Telescope[/ITAL] Observations of PG 1114+445." The Astrophysical Journal 503 (1) (August 10): L23-L26. doi:10.1086/311520.

\section{Published Version}

doi:10.1086/311520

\section{Permanent link}

http://nrs.harvard.edu/urn-3:HUL.InstRepos:30212140

\section{Terms of Use}

This article was downloaded from Harvard University's DASH repository, and is made available under the terms and conditions applicable to Other Posted Material, as set forth at http:// nrs.harvard.edu/urn-3:HUL.InstRepos:dash.current.terms-of-use\#LAA

\section{Share Your Story}

The Harvard community has made this article openly available.

Please share how this access benefits you. Submit a story.

\section{Accessibility}




\title{
DISCOVERY OF ASSOCIATED ABSORPTION LINES IN AN X-RAY WARM ABSORBER: HUBBLE SPACE TELESCOPE ${ }^{1}$ OBSERVATIONS OF PG 1114+445
}

\author{
Smita Mathur, ${ }^{2}$ Belinda Wilkes, and Martin Elvis \\ Harvard-Smithsonian Center for Astrophysics, 60 Garden Street, Cambridge, MA 02138 \\ Received 1998 March 18; accepted 1998 June 10; published 1998 July 17
}

\begin{abstract}
The unified picture of X-ray/UV absorbers offers a unique opportunity to probe the nuclear environment of active galactic nuclei. To test the unified absorber scenario and to understand the physical properties of the absorber, we obtained the first UV spectrum of PG 1114+445 using the Hubble Space Telescope (HST) Faint Object Spectrograph. PG 1114+445 is known to have an X-ray ionized ("warm") absorber, so that UV absorption is predicted. The $H S T$ spectrum clearly shows strong UV absorption lines due to Ly $\alpha, \mathrm{C}$ IV, and $\mathrm{N} v$, blueshifted by $\sim 530 \mathrm{~km} \mathrm{~s}^{-1}$ with respect to the quasar redshift. Since both X-ray and UV absorbers are rare in radio-quiet quasars, these observations argue strongly that the X-ray and UV absorbers are closely physically related, perhaps identical. We place constraints on the parameters of the absorber and conclude that the mass outflow rate is comparable to the accretion rate in PG $1114+445$.
\end{abstract}

Subject headings: quasars: absorption lines — quasars: individual (PG 1114+445) — ultraviolet: galaxies

\section{INTRODUCTION}

The discovery that the $\mathrm{O}$ VII, $\mathrm{O}$ VIII $\mathrm{X}$-ray and $\mathrm{O}$ VI UV absorption in quasar 3C 351 could be readily modeled by a single a X-ray/UV absorber (Mathur et al. 1994) offered a new and unique way to determine the physical conditions of the absorbing material in active galactic nuclei (AGNs). As more $\mathrm{X}$-ray/UV absorbers were found ${ }^{3}$ it became clear that, although they span a wide range of parameter space, they are systematically highly ionized, low density, outflowing, situated outside the broad emission line region, and with high column density. With these properties, the X-ray/UV absorbers delineate a previously unrecognized nuclear component of AGNs with important consequences-a wind or outflow that carries significant kinetic energy and a mass-loss rate comparable to the accretion rate needed to power the AGN continuum (Mathur, Elvis, \& Wilkes 1995; hereafter, MEW95). The X-ray/ UV connection was further strengthened when Crenshaw (1997) found that UV and X-ray absorption tend to occur together in Seyfert 1 galaxies with all of the warm absorber Seyferts showing UV absorption while those without a warm absorber not showing it. ${ }^{4}$ It is possible that a wide range of associated absorbers, including broad absorption line systems, are related through a continuum of properties such as the column density, ionization parameter, and outflow velocity.

First, though, we need to establish how common the X-ray/ UV absorbers are, whether the X-ray absorbers are really physically related to the UV absorbers in all AGNs, and whether this unified picture is quite general or is merely a chance coincidence in a minority of cases. In order to answer these ques-

\footnotetext{
${ }^{1}$ Based on observations with the NASA/ESA Hubble Space Telescope, obtained at the Space Telescope Science Institute, which is operated by the Association of Universities for Research in Astronomy, Inc., under NASA contract NAS5-26555.

2 smita@cfa.harvard.edu.

3 The more recently discovered X-ray/UV absorbers are the "red" quasar 3C 212 (Mathur 1994); a variable Seyfert galaxy NGC 5548 (MEW95); the broad absorption line quasars (BALQSOs) PHL 5200 (Mathur, Elvis, \& Singh 1995); the Seyfert galaxy NGC 3516, explaining the previous presence and present disappearance of its broad absorption lines (Mathur, Wilkes, \& Aldcroft 1997); and the Seyfert galaxy NGC 3783 (Shields \& Hamann 1997).

${ }^{4}$ Mrk 509, which is "No" for having a warm absorber in Crenshaw (1997), is actually a "Yes" (Reynolds 1997).
}

tions as well as to probe the nuclear material in more objects, we initiated a Hubble Space Telescope (HST) program to search quasars known to have X-ray ionized ("warm") absorbers for the UV absorbers predicted by the unified X-ray/UV absorber model. Although common in Seyfert galaxies and radio-loud quasars, UV absorption is rare in radio-quiet quasars (§ 3.1); hence, these observations give a statistically strong test.

Here we present a positive detection for the first object observed: PG 1114+445. This is a radio-quiet quasar at $z=$ 0.144 , and it was the only one of a well-defined subsample of 23 PG quasars to show an ionized absorber signature in its ROSAT spectrum (Laor et al. 1994).

\section{THE HST SPECTRA}

On 1996 November 23, we observed PG $1114+445$ with the Faint Object Spectrograph (FOS) on board the HST, using a $1^{\prime \prime}$ aperture. The source acquisition was done in a four-step sequence to ensure pointing accuracy of $0{ }^{\prime \prime} 12$. Grating G130H was used on the blue side of the detector and $\mathrm{G} 190 \mathrm{H}$ and $\mathrm{G} 270 \mathrm{H}$ on the red side. The total exposure was $12,820 \mathrm{~s}$ on G130H, 4360 s on G190H, and 180 s on G270H. We will focus only on the properties of the absorption lines in this paper. The analysis and discussion of the emission-line properties are deferred to another paper (Wills et al. 1998).

The data were reduced with IRAF using the standard procedure described in the HST data handbook. The data accumulated with different exposures were combined and wavelength calibrated. The final spectra are shown in Figure 1. Strong absorption lines are clearly observed superposed on the emission lines of $\operatorname{Ly} \alpha, \mathrm{N} \mathrm{v}$, and $\mathrm{C}$ IV, as expected from the $\mathrm{X}$-ray/UV models. The absorption lines are much deeper than the continuum level, which implies that the broad emission line region is partially covered by the absorbing material. The models also predict $\mathrm{O}$ VI absorption lines. The G130 spectrum around O VI (observed $\lambda=1183 \AA$ ) has a very low signal-tonoise ratio $(\mathrm{S} / \mathrm{N})$ but does show a marginally detected $\mathrm{O}$ VI absorption line. Absorption in the low ionization line of $\mathrm{Mg}$ II is not detected, again as expected given the high ionization of the X-ray absorber.

The measurement of absorption lines situated within a broad emission line profile is inherently uncertain because the real 

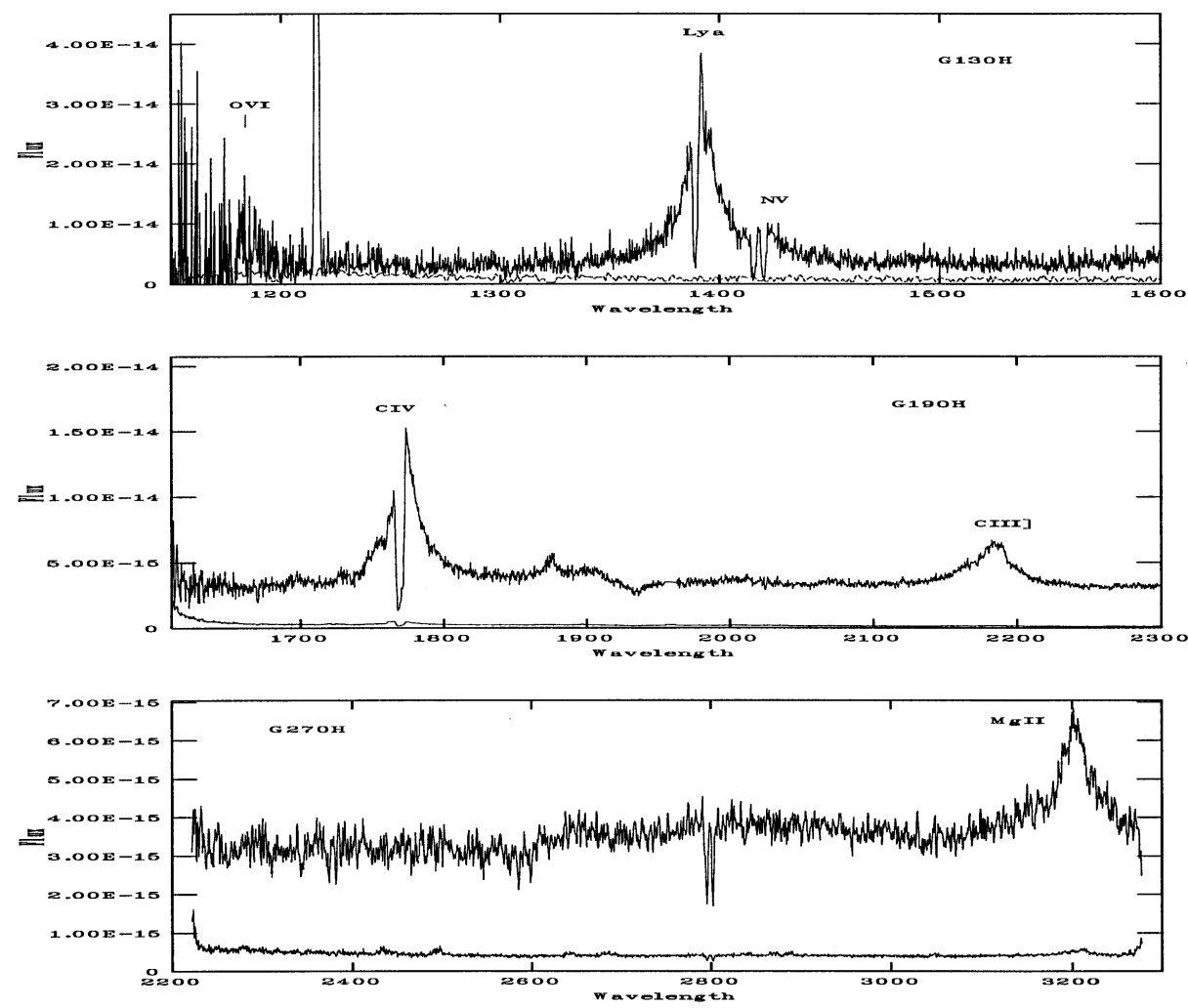

FIG. 1. - HST spectrum of PG 1114+445: absorption lines of $\mathrm{Ly} \alpha, \mathrm{N}$ v, and $\mathrm{C}$ IV are clearly seen. The expected position of $\mathrm{O}$ vI absorption line is marked by a vertical bar. The G270H spectrum is smoothened by two pixels. The lower line in each plot is the error spectrum.

shape of the emission line is unknown. We use two separate methods to measure the line equivalent widths (EWs). The initial analysis of the absorption lines used the IRAF task SPLOT. The EW of the lines was measured by just linear interpolation across the absorption in the emission-line profile, and the results are given in Table 1 . We estimate the SPLOT errors on the EW of the Ly $\alpha$ and C IV lines to be $\sim 40 \%$. The errors on the $\mathrm{N} \mathrm{v}$ doublet are smaller, $\sim 10 \%$, because the $\mathrm{N} \mathrm{V}$ emission line is very weak and so the continuum for the absorption lines is much better defined. The $\mathrm{C}$ IV and $\mathrm{N} v$ doublet components have about the same EW, with doublet ratios close to 1 indicating that the lines are saturated. The troughs do not reach zero intensity; higher resolution observations would be required to detect the dark cores of the lines. It is also possible that the line cores do not reach zero intensity as a result of scattering and/or partial covering (Korista et al. 1992; Barlow, Hamann, \& Sargent 1997). The present observations, however, do not have high enough resolution to detect such effects. The $\mathrm{O}$ VI absorption line is only marginally detected, so we have estimated only a rough lower limit on the EW.

Fitting the emission and absorption lines together in some cases allows better estimates of the EWs of the lines. Accordingly, the STSDAS task SPECFIT (contributed by G. Kriss) was also used to determine line parameters, subject to some assumptions on the shape of the emission-line profile. We characterized the continuum with a simple power law, the emission lines with multiple Gaussians, and each absorption line with a single Gaussian. We first fitted the continuum in featureless parts of the spectrum on either side of the emission/absorption feature to be measured. The parameters of the power-law con-

TABLE 1

Absorption-Line Parameters

\begin{tabular}{|c|c|c|c|c|c|c|c|c|c|c|}
\hline \multirow[b]{2}{*}{ Line, $\lambda$} & \multirow[b]{2}{*}{$\lambda_{\text {obs }}(\AA)$} & \multirow[b]{2}{*}{$z$} & \multicolumn{2}{|c|}{$\mathrm{EW}^{\mathrm{a}}(\AA)$} & \multicolumn{2}{|c|}{ FWHM $\left(\mathrm{km} \mathrm{s}^{-1}\right)$} & \multirow[b]{2}{*}{ OBSERVED $^{\mathrm{b}}$} & \multicolumn{3}{|c|}{ PREDiCTED $N_{\text {ion }}$} \\
\hline & & & SPLOT & SPECFIT & SPLOT & SPECFIT & & $\mathrm{ION}^{\mathrm{c}}$ & CLOUDY $^{\mathrm{d}}$ & $\mathrm{SED}^{\mathrm{e}}$ \\
\hline $\operatorname{Ly} \alpha, 1215.7 \ldots \ldots$ & 1389.1 & $0.1426 \pm 0.0001$ & 1.9 & $3.1 \pm 0.4$ & 500 & $812 \pm 103$ & $>(3-6) \times 10^{14}$ & $3.2 \times 10^{15}$ & $8.9 \times 10^{15}$ & $9.8 \times 10^{15}$ \\
\hline $\mathrm{C}_{\text {IV }}, 1548.2 \ldots \ldots$ & 1768.1 & $0.1420 \pm 0.0005$ & 2.4 & $3.0 \pm 0.1$ & 520 & $590 \pm 14$ & $>(6-7) \times 10^{14}$ & $3.4 \times 10^{14}$ & $1.6 \times 10^{15}$ & $5.0 \times 10^{15}$ \\
\hline C IV, $1550.8 \ldots \ldots$ & 1771.1 & $0.1420 \pm 0.0005$ & 2.1 & $2.5 \pm 0.1$ & 520 & $590 \pm 14$ & & & & \\
\hline $\mathrm{N} \mathrm{v}, 1238.8 \ldots \ldots$ & 1415.7 & $0.1428 \pm 0.0004$ & 1.8 & $2.7 \pm 0.2$ & 470 & $573 \pm 43$ & $>(8-13) \times 10^{14}$ & $3.9 \times 10^{15}$ & $7.1 \times 10^{15}$ & $6.9 \times 10^{16}$ \\
\hline $\mathrm{N} \mathrm{v}, 1242.8 \ldots \ldots$ & 1420.2 & $0.1426 \pm 0.0002$ & 1.9 & $2.4 \pm 0.2$ & 500 & $634 \pm 48$ & & & & \\
\hline O VI, ${ }^{\mathrm{f}} 1033.8 \ldots \ldots$ & 1179 & 0.14 & 2 & & 430 & & $>1 \times 10^{15}$ & $4.5 \times 10^{17}$ & $4.7 \times 10^{17}$ & $3.4 \times 10^{18}$ \\
\hline
\end{tabular}

${ }^{a}$ Rest-frame EW. SPLOT error estimates are $40 \%$ on Ly $\alpha$ and C IV, $10 \%$ on $\mathrm{N} \mathrm{v}$.

b Two numbers correspond to SPLOT and SPECFIT values.

${ }^{\mathrm{c}}$ Netzer 1996 input continuum in photoionization code ION, from George et al. 1997.

"Input continuum as "Table AGN" in photoionization code CLOUDY.

e Spectral energy distribution: CLOUDY Table AGN continuum modified to observed X-ray parameters.

${ }^{\mathrm{f}} \mathrm{O}$ VI absorption line only marginally detected, measurements highly uncertain. 
tinuum, the slope and normalization, were then fixed in subsequent fits. The parameters of the emission lines (flux, centroid, FWHM, and skew) and absorption lines (EW, centroid, and FWHM) were allowed to be free. The fits to the $\operatorname{Ly} \alpha$, $\mathrm{N}$ v, and $\mathrm{C}$ IV profiles are shown in Figure 2, and the corresponding EW and FWHM of the absorption lines are given in Table 1. The two methods, SPLOT and SPECFIT, give consistent results, although the SPECFIT results are systematically higher due to higher effective continuum provided by the emission line. These differences are not important to the current discussion.

The observed FWHM of the lines is 2.3-3.0 $\AA$ ( $\gtrsim 500-600$ $\mathrm{km} \mathrm{s}^{-1}$ ) with SPLOT and SPECFIT, respectively. The lines are quite likely resolved given the nominal spectral resolution of $1-1.5 \AA\left(\sim 200 \mathrm{~km} \mathrm{~s}^{-1}\right)$ in $\mathrm{G} 130 \mathrm{H}$ and $\mathrm{G} 190 \mathrm{H}$ (the FWHM of the Milky Way interstellar Mg II lines is 2-2.2 $\AA$, consistent with the nominal resolution of $2.09 \AA$ in $\mathrm{G} 270 \mathrm{H}$ ). This would imply that the absorber is dispersed in velocity space. The redshift of the absorption lines is 0.142 (Table 1), blueshifted by $530 \mathrm{~km} \mathrm{~s}^{-1}$ compared to the quasar redshift of 0.144 . The redshift of the $\mathrm{O}$ vi line could not be measured accurately due to its very low $\mathrm{S} / \mathrm{N}$ but may be slightly offset to the blue. All the parameters of $\mathrm{O}$ vi given in Table 1 are highly uncertain.

\section{THE X-RAY/UV ABSORBER}

The HST spectrum of PG $1114+445$ shows the associated high-ionization $\operatorname{Ly} \alpha, \mathrm{N}$ v, and $\mathrm{C}$ IV (and possibly O vI) absorption lines predicted from the X-ray/UV models. Is this just a chance coincidence? About $10 \%$ of Seyfert galaxies observed with IUE have associated absorption lines in their UV spectra (Ulrich 1988), so there is a $10 \%$ chance of finding them in any randomly selected Seyfert galaxy. In the HST sample of Seyfert galaxies, Crenshaw (1997) finds the fraction to be $~ 50 \%$. However, for higher luminosity quasars, strong narrow associated absorption appears to arise predominantly in steep spectrum, radio-loud objects (Foltz et al. 1988) rather than in radio-quiet quasars such as PG $1114+445$. In fact, there has been some evidence of a dichotomy between the occurrence of narrow associated absorption in radio-loud quasars and broad associated absorption in radio-quiet quasars (i.e., in BALQSOs; Foltz et al. 1988). Only one of 29 radio-quiet quasars showed associated absorption in the sample studied by Foltz et al.

Similarly, in X-rays, warm absorbers are observed in about half of the Seyfert 1 galaxies (Reynolds 1997). But absorption in radio-quiet quasars is not as frequent. In the complete sample of 20 radio-quiet PG quasars observed by Laor et al. (1994), PG $1114+445$ was the only object with evidence for absorption by ionized matter. The probability for PG $1114+445$ to have associated UV absorption and X-ray ionized absorption by chance is then $\sim 1.7 \times 10^{-3}$. This again strongly suggests that the two absorption systems are physically related.

\subsection{Physical Properties of the Ionized Gas}

A detailed comparison of the UV and X-ray absorbers is required to see if they originate in the same physical component of the nuclear material of the AGN.

The X-ray properties of the ionized absorber in PG $1114+445$, based on ASCA observations, are given in George et al. (1997). The total equivalent hydrogen column density of the PG $1114+445$ absorber is $\sim 2 \times 10^{22} \mathrm{~cm}^{-2}$ and the $\mathrm{X}$-ray ionization parameter $U_{\mathrm{X}}$ is $\sim 0.1$ (corresponding to a
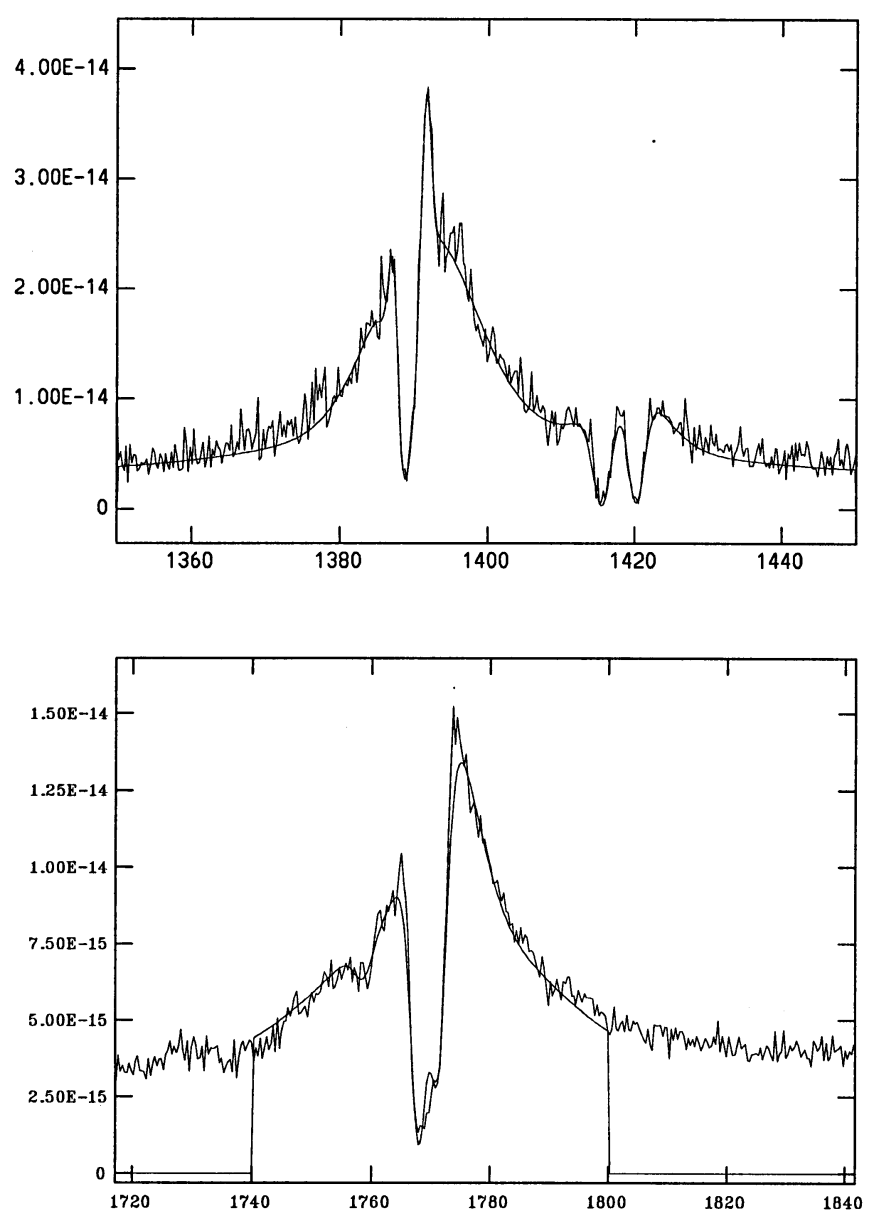

FIG. 2.-SPECFIT fit to the emission and absorption lines (a) Ly $\alpha$ and N $\mathrm{V}$ and $(b) \mathrm{C}$ IV.

traditional ionization parameter $U$ equal to 16.67$).{ }^{5}$ Using the best-fit warm absorber model, George et al. predicted the column densities of the relevant ions using the photoionization code ION (Table 1). The shape of the input continuum used in the ION code is defined in Netzer (1996). The shape of the input continuum can affect the ionization state of the gas considerably (MEW95). So we also used two other ionizing continua as input to the photoionization code CLOUDY (Ferland 1996) to derive the column densities of the UV-observed ions as constrained by the optical depths of the $\mathrm{O}$ VII/O VIII edges observed in ASCA data. Solar abundances are assumed. If we use the standard "Table AGN" continuum (Mathews \& Ferland 1987) given in CLOUDY (corresponding to $U=4.9$ ), the derived column densities are 2-5 times larger (Table 1). The observed continuum of PG 1114+445 itself is somewhat different again. In the CLOUDY Table AGN continuum the Xray power law of slope $\left(f_{\nu} \propto \nu^{-\alpha}\right) \alpha=0.7$ extends down to 0.3 $\mathrm{keV}$, while below $0.3 \mathrm{keV}$ there is a steep upturn into the EUV region. The observed X-ray power-law index in PG 1114+445 is 0.8 all the way down to $0.11 \mathrm{keV}$. The modified Table AGN continuum modified accordingly (corresponding to $U=6.8$ ) gives larger UV line column densities (Table 1) by a factor of 3-10 compared to the Table AGN continuum. Clearly, the ionic

\footnotetext{
${ }^{5} U$ is defined as the dimensionless ratio of ionizing photon to hydrogen number density. Photons with energy larger than 1 ryd are integrated. In $U_{\mathrm{X}}$, only X-ray photons between 0.1 and $10 \mathrm{keV}$ are integrated (Netzer 1996).
} 
column densities are sensitive to the shape of the ionizing continuum.

To compare the UV and X-ray absorbers, we now have to convert the EWs of the absorption lines in the HST spectra to the column densities of the ions. However, as discussed in $\S 2$, the lines are saturated and so column densities cannot be measured directly without knowing the velocity dispersion parameter ( $b$ parameter) of the lines. If the lines are resolved, then $b \sim 360 \mathrm{~km} \mathrm{~s}^{-1}$ [where $b=\mathrm{FWHM} /(4 \ln 2)^{1 / 2}$ ]. For this $b$, the UV and X-ray values of $N_{\mathrm{C} \text { Iv }}$ and $N_{\mathrm{Nv}}$ are in agreement. $N_{\mathrm{H} \text {, }}$ predicted by X-ray models is in fact larger than the UV value. A larger value of $N_{\mathrm{H}}$ in a warm absorber model is also seen in NGC 3783 (Shields \& Hamann 1997), where it is attributed to uncertainties in local continuum placement around Ly $\alpha$ or to higher heavy element abundances. The same possibilities apply to PG $1114+445$. If, however, the lines are not fully resolved (since the resolution is at best half of the observed absorption line width), the $b$ is constrained to be $300 \leqq b \lesssim 400 \mathrm{~km} \mathrm{~s}^{-1}$ for the UV and X-ray values to match. The quantitative agreement of the UV and X-ray derived ion column densities implies that, at a minimum, the X-ray absorber makes a large contribution to the absorption seen in the UV and allows that the UV and X-ray absorption is likely to originate in the same material.

From the blueshifts of the absorption lines, the outflow velocity of the X-ray/UV absorber is about $530 \mathrm{~km} \mathrm{~s}^{-1}$. Outflows are normal in associated absorption systems and can be used to derive some interesting numbers (MEW95) assuming that the UV and X-ray absorbers are the same. We know that the absorber has a column density of $N_{\mathrm{H}}=2 \times 10^{22}$ atoms $\mathrm{cm}^{-2}$ and ionization parameter $U=4.9$ (for the CLOUDY Table AGN continuum). From photoionization modeling, the distance of the absorber from the nucleus is $r=2.5 \times 10^{18} n_{5}^{-1 / 2} \mathrm{~cm}$ (where $n_{5}$ is density in the units of $10^{5} \mathrm{~cm}^{-3}$ ). Similar to other $\mathrm{X}$-ray/UV absorbers $(\S 1)$, the depth of the absorption lines is larger than the continuum level (Fig. 1), so the absorber must cover a substantial fraction of the broad emission line region (BELR). It may be situated outside the BELR or it could also be cospatial with it if most of the emission is beamed from the far side of the BELR. Scaling from the BELR size from the reverberation mapping of NGC 5548 (Clavel et al. 1991) for PG $1114+445$ luminosity $\left(L_{\mathrm{Bol}}=5 \times 10^{45} \mathrm{ergs} \mathrm{s}^{-1}\right)$ yields a distance of the BELR from the central continuum of $3.7 \times$ $10^{17} \mathrm{~cm}$. So the distance of the absorber, $r$, is $\gtrsim 3.7 \times 10^{17} \mathrm{~cm}$. This puts an upper limit on the density: $n<43 n_{5} \mathrm{~cm}^{-3}$. The mass of the absorber is then $130 f_{0.1} n_{5}^{-1} M_{\odot}$ for a covering factor of $f=10 \%$. The line-of-sight mass outflow rate would be $\dot{M}_{\text {out }}=1 f_{0.1} M_{\odot} \mathrm{yr}^{-1}$, comparable to the accretion rate of 0.9 $M_{\odot} \mathrm{yr}^{-1}$ needed to power PG $1114+445$ at $10 \%$ efficiency. The line-of-sight kinetic energy carried out in the flow is not as large, however, $\sim 10^{41} \mathrm{ergs} \mathrm{s}^{-1}$ (cf. $\dot{M}_{\text {out }}=10 f_{0.1} M_{\odot} \mathrm{yr}^{-1}$ in NGC 5548 and a kinetic energy of $\sim 10^{43} \mathrm{ergs} \mathrm{s}^{-1}, L_{\mathrm{Bol}}=$ $5 \times 10^{44}$ ergs s$^{-1}$ ).

\section{CONCLUSIONS}

The HST FOS UV spectrum of PG $1114+445$ contains associated high-ionization, UV absorption lines, as predicted from models of the X-ray ionized absorber. We find that the absorber is situated outside the BELR or cospatial with it and outflowing with a line-of-sight velocity of $\sim 530 \mathrm{~km} \mathrm{~s}^{-1}$. The mass outflow rate of $1 M_{\odot} \mathrm{yr}^{-1}$ for a $10 \%$ covering factor is comparable to the accretion rate onto the nuclear black hole.

The rarity of both UV and X-ray absorbers individually in radio-quiet quasars virtually requires that the $\mathrm{X}$-ray and UV absorbers are closely physically related. The consistency of the column densities obtained from both UV and X-ray data suggests that they are perhaps identical. At a minimum, the X-ray absorber makes a substantial contribution to the absorption seen in the UV. Thus, the absorber in PG $1114+445$ satisfies both statistical as well as physical tests of our X-ray/UV absorber model.

We would like to thank the referee, Kirk Korista, for very useful comments, Ian George for discussions and an early copy of his paper, and Ian Evans for discussions regarding the FOS calibration. S. M. is supported through NASA grants NAG53249 (LTSA) and GO-06484.01-95A from Space Telescope Science Institute. B. J. W. is supported through NASA contract NAS8-39073 (ASC).

\section{REFERENCES}

Barlow, T. A., Hamann, F., \& Sargent, W. L. W. 1997, in ASP Conf. Ser. 128, Mass Ejection from AGN, ed. N. Arav, I. Shlosman, \& R. J. Weymann (San Francisco: ASP), 13

Clavel, J., et al. 1991, ApJ, 366, 64

Crenshaw, M. 1997, in ASP Conf. Ser. 128, Mass Ejection from AGN, ed. N. Arav, I. Shlosman, \& R. J. Weymann (San Francisco: ASP), 121

Ferland, G. 1996, Univ. Kentucky, Department of Physics and Astronomy Internal Report

Foltz, C. B., Chaffee, F. H., Weymann, R. J., \& Anderson, S. F. 1988, in QSO Absorption Lines, ed. J. C. Blades, D. Turnshek, \& C. Norman (Cambridge: Cambridge Univ. Press), 53

George, I. M., et al. 1997, ApJ, 491, 508

Korista, K., et al. 1992, ApJ, 401, 529
Laor, A., Fiore, F., Elvis, M., Wilkes, B., \& McDowell, J. 1994, ApJ, 435, 611

Mathews, W. G., \& Ferland, G. J. 1987, ApJ, 323, 456

Mathur, S. 1994, ApJ, 431, L75

Mathur, S., Elvis, M., \& Singh, K. P. 1995, ApJ, 455, L9

Mathur, S., Elvis, M. S., \& Wilkes, B. J. 1995, ApJ, 452, 230 (MEW95)

Mathur, S., Wilkes, B. J., \& Aldcroft, T. 1997, ApJ, 478, 182

Mathur, S., Wilkes, B. J., Elvis, M. S., \& Fiore, F. 1994, ApJ, 434, 493

Netzer, H. 1996, ApJ, 473, 781

Reynolds, C. S. 1997, MNRAS, 286, 513

Shields, J., \& Hamann, F. 1997, ApJ, 481, 752

Ulrich, M. H. 1988, MNRAS, 230, 121

Wills, B., et al. 1998, in preparation 